\title{
Decomposition of a class of linear electrical networks for calculation of total power
}

\author{
MILOVAN RADULOVIĆ ${ }^{1, *}$, TOMISLAV B ŠEKARA ${ }^{2}$ and BUDIMIR LUTOVAC ${ }^{1}$ \\ ${ }^{1}$ Faculty of Electrical Engineering, University of Montenegro, Cetinjski put bb, 81000 Podgorica, Montenegro \\ ${ }^{2}$ School of Electrical Engineering, University of Belgrade, Bulevar kralja Aleksandra 73, Belgrade 11000, \\ Serbia \\ e-mail: milovanr@ac.me; tomi@etf.rs; budo@ac.me
}

MS received 5 December 2016; revised 18 December 2017; accepted 26 February 2018; published online 17 July 2018

\begin{abstract}
A new method of decomposition of linear electrical networks for calculating total power is presented. By an iterative procedure, on the basis of decomposition of the electrical network, the branches containing power sources (voltage and/or current) are selected first until the remaining network becomes passive. Then, one calculates the power dissipated by the decomposed networks supplied by the corresponding power sources. Total power dissipated by impedances of the starting networks is equal to the sum of powers dissipated by impedances of the decomposed networks, under the condition that all impedances $Z$ of the network are either resistive $\operatorname{Im}(Z)=0$ or reactive $\operatorname{Re}(Z)=0$ or mutually equal.
\end{abstract}

Keywords. Decomposition; iterative procedure; linear electrical network; Tellegen's theorem.

\section{Introduction}

Tellegen's theorem is a consequence of Kirchoff's law; therefore, it can be applied to electrical systems, either linear or nonlinear, reciprocal or nonreciprocal, time varying or time invariant [1-3]. Tellegen's theorem has also a significant application in performing the analyses of sensitivities of electrical networks, including electric power systems [4]. The use of variational principle for power system analysis is explained in [5], while several methods for calculation of power based on decomposition of electrical networks may be found in [6-9]. This work presents a new method of calculation of total power of the source by the method of decomposition or the total power dissipated in impedances in a linear electrical network (LEN), under the condition that all impedances $Z$ of the network are either resistive $\operatorname{Im}(Z)=0$ or reactive $\operatorname{Re}(Z)=0$ or mutually equal. In performing these calculations in complex LENs, one applies either the basic or other methods derived through application of various transforms, either exact or approximative [1-3].

The significance of power calculations in electrical networks concerns primarily the analyses of electrical energy savings accomplished by application of suitable optimization techniques. In this paper a new simple method for calculating total power in a complex electrical network, supplied by voltage and/or current sources [10-12], based on the decomposition is presented.

*For correspondence

\section{Decomposition of LEN supplied by voltage sources}

Let us formulate the theorem concerning total power dissipated by impedances in a LEN of harmonic current supplied by voltage sources, i.e., the total complex power of the source.

Theorem 1 (Decomposition of LEN supplied by voltage sources) Let a given LEN $N_{0}$ consist of $n$ branches conducting sinusoidal currents, as shown in figure 1. By decomposition a new unconnected network $N$, shown in figure 2, consisting of two networks $N_{1}$ and $N_{T}$ is formed. Network $N_{1}$ represents network $N_{0}$ having jth branch disconnected and network $N_{T}$ is Thevenin's circuit of jth branch of network $N_{0}$. Then, total complex power of the voltage sources of network $N_{0}, S_{E, N_{0}}$, under condition that all network impedances $Z$ are either resistive $\operatorname{Im}(Z)=0$ or reactive $\operatorname{Re}(Z)=0$ or mutually equal, is equal to total power of the sources of unconnected network $N, S_{E, N}$ :

$$
S_{E, N_{0}}=S_{E, N}
$$

Proof Let us write total complex power of the sources of the unconnected network $N$ in the following form:

$$
S_{E, N}=S_{E, N_{1}}+S_{E, N_{T}}
$$

where $S_{E, N_{1}}$ is the total complex power of the sources of network $N_{1}$ and $S_{E, N_{T}}$ is the total complex power of the sources of network $N_{T}$; in the developed form, on the basis of figure 2 


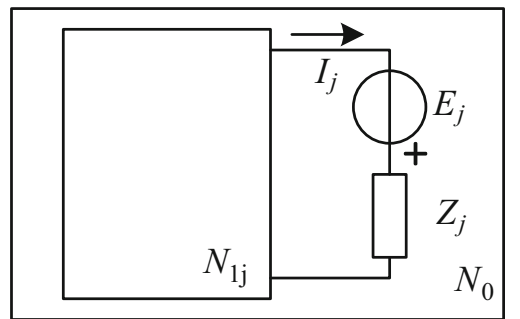

Figure 1. Electrical network $N_{0}$ consisting of a separate complex branch $j$ and network $N_{1 j}$.

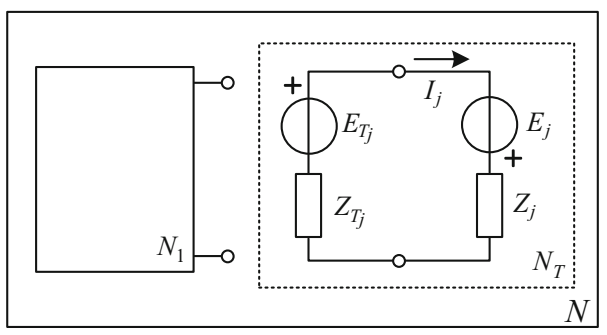

Figure 2. Network $N$ as an equivalent to its decomposition to networks $N_{1}$ and $N_{T}$ as regards the total complex power source.

$$
\begin{gathered}
S_{E, N_{1}}=\sum_{\substack{k=1 \\
k \neq j}}^{n} E_{k}(\underbrace{I_{k}+\Delta I_{k}}_{I^{\prime} k})^{*}, \\
S_{E, N_{T}}=E_{T j} I_{j}^{*}+E_{j} I_{j}^{*},
\end{gathered}
$$

$I_{k}$ is the branch current of network $N_{0}, k=\overline{1, n}$,

$I_{k}^{\prime}$ is the branch current of network $N_{1}, k=\overline{1, n}, k \neq j$ upon disconnection of the $j$ th branch of network $N_{0}$ (change of parameters of branch $j$ ) and superscript $*$ denotes conjugate value.

$\Delta I_{k}$ is the increment (change) of current in branch $k=$ $\overline{1, n}$ upon change of parameters of network $N_{0}$.

Using relations (2), (3) and (4), the complex power of the source of unconnected network $N$ is obtained in the form

$$
S_{E, N}=\underbrace{\sum_{k=1}^{n} E_{k} I_{k}^{*}}_{S_{E, N_{0}}}+\underbrace{\sum_{\substack{k=1 \\ k \neq j}}^{n} E_{k} \Delta I_{k}^{*}+E_{T j} I_{j}^{*}}_{A} .
$$

If we prove that $A=0$, the proof of theorem 1 follows from relation (5). It is required to prove that

$$
A=\sum_{\substack{k=1 \\ k \neq j}}^{n} E_{k} \Delta I_{k}^{*}+E_{T j} I_{j}^{*}=0 .
$$

Let us quote theorem of variation together with its derivation [2, 3], which will be used to prove relation (6). For the sake of transparency and simplicity of tracking, using the present notation, with no loss of generality, let us restrict to branch $j$ where a variation of impedance occurred. Let the change of impedance of $j$ th branch amount to $\Delta Z_{j}$; then, according to the theorem of compensation, this change of impedance causes a change of the distribution of currents in the $j$ th branch, which would have been obtained by the change of electromotive force of $j$ th branch:

$$
\Delta E_{j}=-\Delta Z_{j} I_{j}^{\prime}
$$

where $I_{j}^{\prime}$ is the current of branch $j$, which corresponds to the current distribution in the network after the change of impedance in branch $j$ :

$$
I_{j}^{\prime}=I_{j}+\Delta I_{j}
$$

According to the principle of superposition, current in branch $j$ is equal to the sum of current $I_{j}$ (current prior to variation $\Delta Z_{j}$ ) due to all $k$ sources $E_{k}$ of network $N_{0}$ and source $\Delta E_{j}$ in relation (7); thus, one may write

$$
I_{j}+\Delta I_{j}=I_{j}+y_{j j} \Delta E_{j} .
$$

From relations (7), (8) and (9) one obtains

$$
\Delta I_{j}=-\frac{y_{j j} \Delta Z_{j}}{1+y_{j j} \Delta Z_{j}} I_{j},
$$

where $y_{j j}$ is the input admittance of branch $j$ with respect to voltage source $E_{j}$ in branch $j$. Input admittance $y_{j j}$ can be determined from the relation

$$
y_{j j}=\frac{I_{j}}{E_{j}} \text { for } E_{k}=0, k \neq j .
$$

From the property of linearity of network $N_{0}$, the following relation concerning the change of current $\Delta I_{k}$ in branch $k$ due to a change of current $\Delta I_{j}$ in branch $j$ clearly follows:

$$
\Delta I_{k}=\frac{y_{k j}}{y_{j j}} \Delta I_{j}
$$

where $y_{k j}$ is the transfer admittance of branch $k$ with respect to voltage source $E_{j}$ in branch $j$. Transfer admittance $y_{k j}$ can be determined from the relation

$$
y_{k j}=\frac{I_{k}}{E_{j}} \text { for } E_{k}=0, k \neq j .
$$

Finally, using relations (10) and (12) one obtains the law of change of current $\Delta I_{k}$ with variation of impedance $\Delta Z_{j}$ :

$$
\Delta I_{k}=-\frac{y_{k j} \Delta Z_{j}}{1+y_{j j} \Delta Z_{j}} I_{j}
$$

This demonstrates the theorem of parameter variation. In order to prove theorem 1 , consider LEN $N_{0}$, figure 3 , for $E_{g}=0$ and $g \neq k, j$, which corresponds to network $N_{1}$ with the $j$ th branch separated. 
Determine now the input $y_{i j}$ and transfer admittance $y_{k j}$ defined previously via reducing down the problem to input admittance $y_{j j}^{\prime}$ and transfer admittance $y_{k j}^{\prime}$ of network $N_{1}^{\prime}$. Since, by definition and using admittance parameters [10], for network $N_{1}^{\prime}$ with $E_{g}=0, g \neq k, j$ one can write exact relations

$$
\begin{gathered}
I_{k}=y_{k k}^{\prime} E_{k}-y_{k j}^{\prime} U_{j}, \\
I_{j}=y_{j k}^{\prime} E_{k}-y_{j j}^{\prime} U_{j}, \\
y_{k j}^{\prime}=y_{j k}^{\prime},
\end{gathered}
$$

based on figure 3 and relations (15)-(17), it is possible to express $I_{k}$ as a function of $E_{j}$ by the following relation:

$$
I_{k}=y_{k j}^{\prime} \frac{Z_{T_{j}}}{Z_{T_{j}}+Z_{j}} E_{j}=y_{k j}^{\prime} \frac{1}{1+y_{j j}^{\prime} Z_{j}} E_{j}, E_{k}=0, k \neq j .
$$

Thus, we obtain $y_{k j}$ via $y_{k j}^{\prime}$ since $y_{j j}^{\prime}=1 / Z_{T_{j}}$ is input admittance of network $N_{1}^{\prime}$ with respect to branch $j$ :

$$
y_{k j}=y_{k j}^{\prime} \frac{1}{1+y_{j j}^{\prime} Z_{j}} .
$$

Analogously, it is straightforward to show that input admittance is

$$
y_{j j}=\frac{1}{Z_{T_{j}}+Z_{j}}=y_{j j}^{\prime} \frac{1}{1+y_{j j}^{\prime} Z_{j}} .
$$

Of interest for this proof is the ratio of transfer admittance $y_{k j}$ to input admittance $y_{j j}$, i.e.

$$
\frac{y_{k j}}{y_{j j}}=\frac{y_{k j}^{\prime}}{y_{j j}^{\prime}} .
$$

Using expression (14), for a variation of impedance of $j$ th branch $\Delta Z_{j} \rightarrow \infty$ one easily obtains increment of current in $k$ th branch:

$$
\Delta I_{k}=-\frac{y^{\prime}{ }_{k j}}{y_{j j}^{\prime}} I_{j}
$$

Since mapping of the increment of current $\Delta I_{j}$ of $j$ th branch to the increment of current $\Delta I_{k}$ of $k$ th branch is in

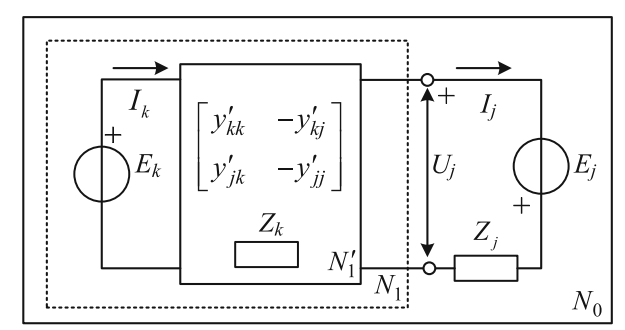

Figure 3. Linear electrical network $N_{0}$ for $E_{g}=0$ and $g \neq k, j$. question, the same mapping of the conjugated values of the current applies; therefore, one can write the relation

$$
\Delta I_{k}^{*}=-\left(\frac{y^{\prime}{ }_{k j}}{y_{j j}^{\prime}}\right)^{*} I_{j}^{*} .
$$

Introducing relation (23) in relation (6) one obtains

$A=I_{j}^{*}\left(-\sum_{\substack{k=1 \\ k \neq j}}^{n}\left(\frac{y_{k j}^{\prime}}{y_{j j}^{\prime}}\right)^{*} E_{k}+E_{T_{j}}\right)=0$ for $\left(\frac{y^{\prime}{ }_{k j}}{y_{j j}^{\prime}}\right)^{*}=\frac{y^{\prime}{ }_{k j}}{y_{j j}^{\prime}}$.

Since the disconnected $j$ th branch is in question, value of the current is $I_{j}^{\prime}=0$ and $\left(y^{\prime}{ }_{k j} / y_{j j}^{\prime}\right)^{*}=y_{k j}^{\prime} / y_{j j}^{\prime}$. Since parameters of network $N_{1}^{\prime}$ are not dependent upon excitation, it clearly follows from relations (16) and (17) that

$$
\sum_{\substack{k=1 \\ k \neq j}}^{n} \frac{y_{k j}^{\prime}}{y_{j j}^{\prime}} E_{k}=E_{T_{j}},
$$

which represents known equality of superposition of the sources of each $k$ th branch at the place of disconnection of $j$ th branch, i.e., Thevenin's source $E_{T_{j}}$. Alternatively, if $I_{j}^{*}=0$, there follows a trivial solution of relation (24). Condition $\left(y^{\prime}{ }_{k j} / y_{j j}^{\prime}\right)^{*}=y_{k j}^{\prime} / y_{j j}^{\prime}$ is fulfilled when all impedances $Z$ of the network are either resistive $\operatorname{Im}(Z)=0$ or reactive $\operatorname{Re}(Z)=0$ or mutually equal. This is a complete proof of theorem 1.

On the basis of theorem 1, by continuing decomposition of network $N_{1}$, in the way of decomposition of network $N_{0}$, until simple circuit $N_{n-1}$ is obtained, it clearly follows that total power of the sources expressed in the form of the sum of all powers of the sources obtained by Thevenin's circuits, as shown in figure 2 in the part of the non-connected network $N$, i.e., iterative application of Tellegen's theorem under the condition that all impedances $Z$ of the network are either resistive $\operatorname{Im}(Z)=0$ or reactive $\operatorname{Re}(Z)=0$ or mutually equal:

$$
S_{E, N_{0}}=\sum_{k=1}^{n-1}\left(E_{T_{k}}+E_{k}\right) I_{k}^{*}
$$

\section{Decomposition of LENs comprising current sources}

In order to determine total power dissipated in impedances of a network comprising current sources, i.e., total complex source power, we shall, as the basis, use the Norton 
theorem $[2,3]$ for the application and formulation of theorem 2, which is dual to the previous theorem 1; therefore, it will not be proved separately.

Theorem 2 (Decomposition of LEN comprising current sources) Let there be given a LEN $K_{0}$ consisting of $n$ branches conducting sinusoidal currents, as shown in figure 4. By decomposition a new unconnected network $K$, shown in figure 5, consisting of two networks $K_{1}$ and $K_{T}$ is formed. Network $K_{1}$ represents network $K_{0}$ having jth branch disconnected and network $K_{N}$ is Norton's circuit of jth branch of network $K_{0}$. Then, total complex power of the current sources of network $K_{0}, S_{I, K_{0}}$, under the condition that all network impedances $Z$ are either resistive $\operatorname{Im}(Z)=$ 0 or reactive $\operatorname{Re}(Z)=0$ or mutually equal, is equal to total power of the sources of unconnected network $N, S_{I, K}$, i.e.

$$
S_{I, K_{0}}=S_{I, K}
$$

On the basis of theorem 2, by continuing further decomposition of network $K_{1}$, like decomposition of $K_{0}$, until simple electrical circuit $K_{n-1}$ is obtained, it clearly follows that total power of the sources is expressed in the form of the sum of all powers of the sources obtained by Norton's circuits, as shown in figure 5. Iterative application of the proposed decomposition method, in the part of the non-connected network $K$, under the condition that all impedances $Z$ of the network are either resistive $\operatorname{Im}(Z)=0$ or reactive $\operatorname{Re}(Z)=0$ or mutually equal, is

$$
S_{I, K_{0}}=\sum_{k=1}^{n-1} U_{k}\left(I_{k}-I_{N_{k}}\right)^{*} .
$$

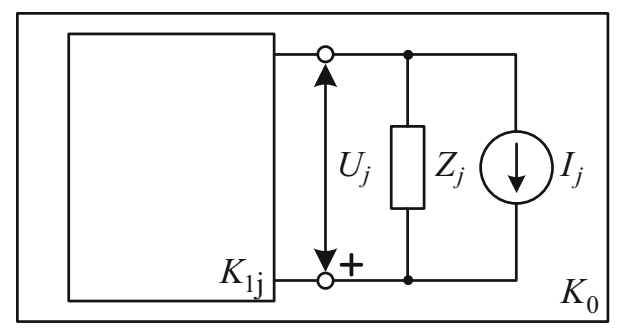

Figure 4. Electrical network $K_{0}$ consisting of a separate complex branch $j$ and network $K_{1 j}$.

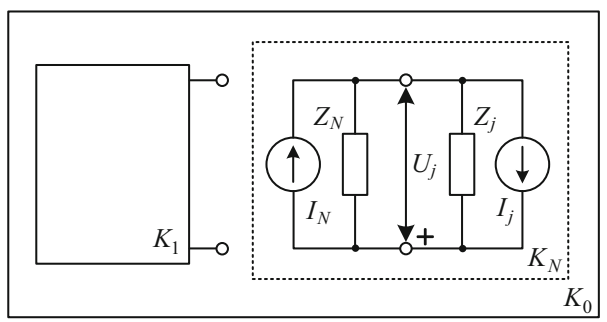

Figure 5. Network as an equivalent to its decomposition to networks $K_{1}$ and $K_{N}$ as regards the total complex power source.
Corollaries of theorem 1 and theorem 2. In order to obtain as low as possible number of the simple circuits created by decomposition of a network supplied by voltage/ current sources, if there are branches containing no voltage/ current source, the strategy should be such that active branches (branch containing a voltage/current source) are disconnected first. In this way the number of iterative decompositions $M_{g}$ (number of active branches) is reduced. Analogously, the number of independent contours $M_{c}$ in a given network supplied by voltage sources is equal to the number of iterations in the process of disconnecting a branch belonging to an independent contour. The number of required iterations $M_{I}$ will be

$$
M_{I}<\min \left[M_{g}, M_{c}\right]<n-1 .
$$

\section{Decomposition of linear complex electrical network}

In order to determine total power dissipated in impedances of a complex network $C_{0}$ (a network supplied by both voltage and current sources) the property of decomposition of a complex network $C_{0}$ to a network supplied by voltage sources $N_{0}$ and a network supplied by current sources $K_{0}$, as shown in figure 6 , is applied first. For these networks the following rule applies [3]:

$$
S_{G, C_{0}}=S_{E, N_{0}}+S_{I, K_{0}}
$$

where $S_{G, C_{0}}$ is the complex power of all sources (voltage and current) in a complex network, $S_{E, N_{0}}$ is the complex power in a network supplied by voltage sources and $S_{I, K_{0}}$ is the complex power in a network supplied by current sources. In the second step, theorems 1 and 2, i.e., iterative application of the proposed decomposition method to the decomposed network supplied by voltage sources and the decomposed network supplied by current sources, are applied. This completes the formulation of application of decomposition of a LEN for the purpose of application of Tellegen's theorem. Using the method described earlier, further investigations would refer to the adequate compensation as well as the definition of power [13-16] on certain parts of the network in power systems.

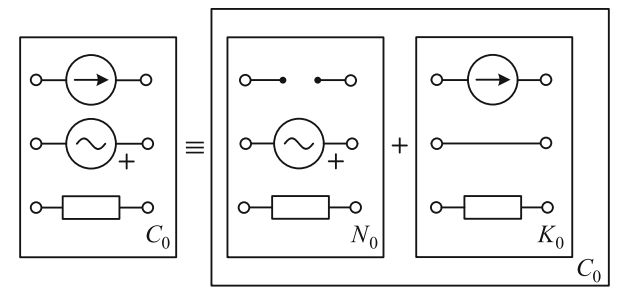

Figure 6. Network $C_{0}$ as an equivalent to its decomposition to networks $N_{0}$ and $K_{0}$ as regards the total complex power source. 


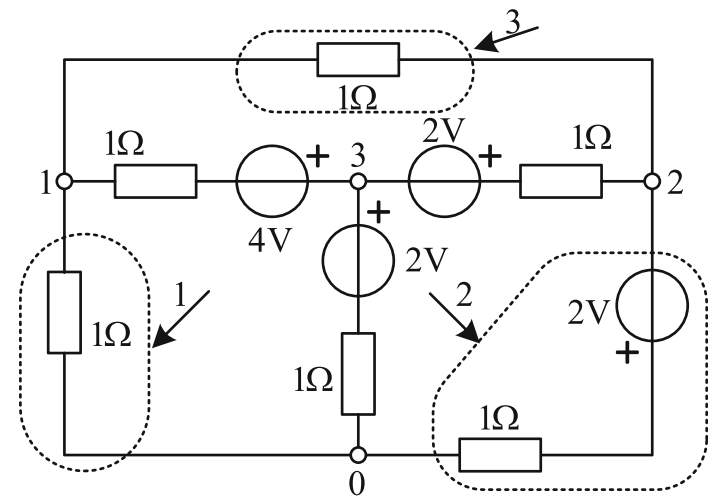

Figure 7. DC electrical network supplied by voltage sources.

Example 1 For a DC network supplied by voltage sources, shown in figure 7 , determine total power dissipated by all resistors in the network by applying decomposition of a linear network for the purpose of iterative application of Tellegen's theorem.

Using the previously described method, for decomposition of linear network of figure 7, e.g., branch 1, figure 8a shows the equivalent Thevenin's circuit $[2,3]$. On the basis of the rule for calculating Thevenin's voltage it follows that $E_{T_{1}}=-U_{10}=2[\mathrm{~V}]$. Power of $E_{T_{1}}$ source is $P_{E, N_{T_{1}}}=2[\mathrm{~W}]$.

Now, branch 2 is disconnected, in accordance with theorem 1, and in a similar way one obtains $U_{20}=E_{T_{2}}=$ $2[\mathrm{~V}]$ and $R_{T_{2}}=5 / 3[\Omega]$; therefore, a new Thevenin's circuit, shown in figure $8 \mathrm{~b}$, is obtained. It is obvious that total power of Thevenin's circuit $N_{T_{2}}$ is $P_{E, N_{T_{2}}}=6[\mathrm{~W}]$. Finally, for the simple circuit $N_{T_{3}}$, shown in figure $8 \mathrm{c}$, power of the source is easily calculated as $P_{E, N_{T_{3}}}=12[\mathrm{~W}]$. If now all obtained powers of the simple Thevenin's circuits are added together, one obtains total source power of the network of figure 7, i.e.

$$
P_{E, N_{T}}=P_{E, N_{T_{1}}}+P_{E, N_{T_{2}}}+P_{E, N_{T_{3}}}=20[\mathrm{~W}] .
$$

Example 2 For AC network supplied by voltage and current sources, shown in figure 9, determine total apparent power $S$ by applying decomposition of a LEN for the purpose of applying Tellegen's theorem. Values of the elements are $E_{1}=(10+\mathrm{j} 20)[\mathrm{V}], E_{3}=(3-\mathrm{j} 11)[\mathrm{V}], E_{5}=(-3+\mathrm{j} 11)$ $[\mathrm{V}], \quad I_{g}=\mathrm{j} 0.5 \quad[\mathrm{~A}], \quad Z_{1}=\mathrm{j} 100[\Omega], \quad Z_{2}=-\mathrm{j} 10[\Omega]$, $Z_{3}=\mathrm{j} 20[\Omega], Z_{4}=\mathrm{j} 20[\Omega]$ and $Z_{5}=-\mathrm{j} 40[\Omega]$.

For a network supplied by both voltage and current sources, the network supplied exclusively by current sources (figure 10a), voltage sources are represented by short circuits, is analysed first and then the network supplied exclusively by voltage sources (figure 10b), current sources are disconnected, is analysed.

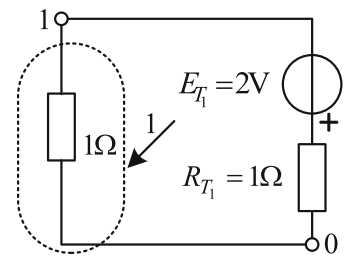

(a)

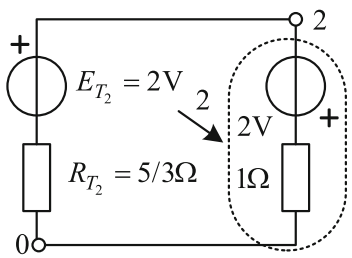

(b)

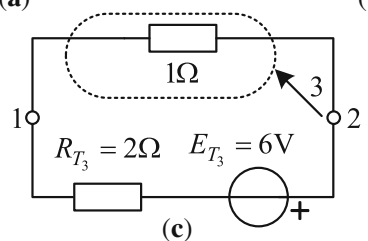

(c)

Figure 8. A decomposition of linear network of figure 7 .

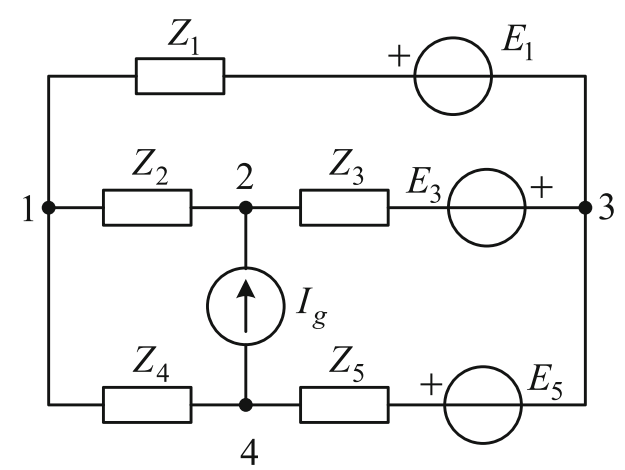

Figure 9. AC electrical network supplied by voltage and current sources.

A decomposition of these networks is shown in figure 11. Using the decomposition method as in the previous example, for the network supplied exclusively by current sources one obtains $Z_{T_{2}}=\mathrm{j} 20[\Omega]$, as shown in figure 11a. The apparent power is now $S_{E, N_{T_{1}}}=Z_{T_{1}} I_{g} I_{g}^{*}=\mathrm{j} 5[\mathrm{VA}]$. For the network supplied exclusively by voltage sources by disconnecting, e.g., branches accommodating impedances $Z_{4}, Z_{5}$ and source $E_{5}$, the equivalent Thevenin's circuit is shown in figure $11 \mathrm{~b}$. On the basis of the rule for calculating Thevenin's voltage it follows that $E_{T_{2}}=(-1.8182+\mathrm{j} 11.8182)[\mathrm{V}], \quad Z_{T_{2}}=$ $\mathrm{j} 9.0909[\Omega]$ and $I_{N_{2}}=(0.0750-\mathrm{j} 0.1083)[\mathrm{A}]$. The apparent power is now $S_{E, N_{T_{2}}}=E_{5} I_{N_{2}}^{*}-E_{T_{2}} I_{N_{2}}^{*}=-\mathrm{j} 0.1894[\mathrm{VA}]$. By disconnecting this branch, the remaining circuit is shown in figure 11c. Now, $I_{N_{3}}=(0.0818-j 0.1182)[\mathrm{A}]$ and the apparent complex power is $S_{E, N_{T_{3}}}=E_{3} I_{N_{3}}^{*}+$ $E_{1} I_{N_{3}}^{*}=\mathrm{j} 2.2727$ [VA]. Adding the obtained powers of the sources of the simple Thevenin's circuits together, the total source power of the network of figure 11 is

$$
\begin{gathered}
S_{E, N_{T}}=S_{E, N_{T_{1}}}+S_{E, N_{T_{2}}}+S_{E, N_{T_{3}}} \\
=\mathrm{j} 5-\mathrm{j} 0.1894+\mathrm{j} 2.2727=\mathrm{j} 7.0833[\mathrm{VA}] .
\end{gathered}
$$

Solving the initial circuit shown in figure 9 , one obtains that currents in the branches containing power sources as 


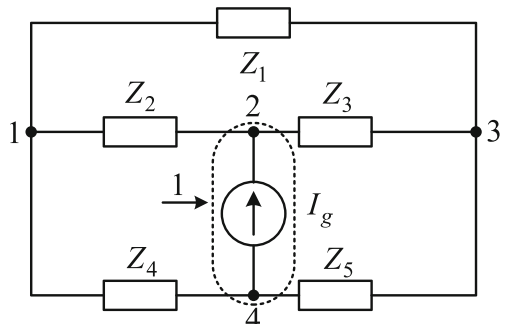

(a)

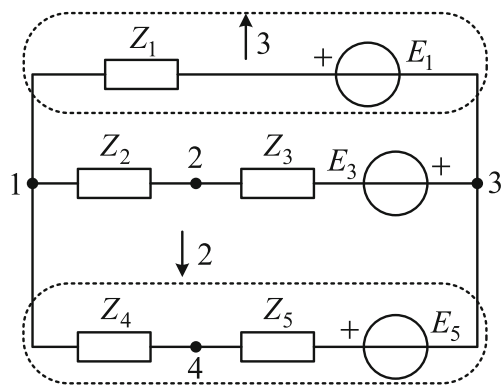

(b)

Figure 10. Network supplied by (a) current sources only and (b) voltage sources only.
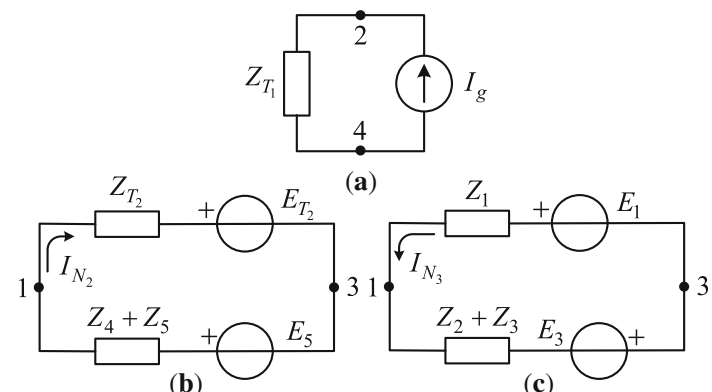

Figure 11. A decomposition of AC networks of figure 10.

follows: $\quad I_{3}=(0.1500-\mathrm{j} 0.7167)[\mathrm{A}], \quad I_{1}=(0.0750-$ $j 0.1083)[\mathrm{A}]$ and $I_{5}=(0.0750-\mathrm{j} 0.6083)[\mathrm{A}]$, while the voltage is $U_{24}=-10$ [V]. The total apparent source power is

$$
S_{E, I}=E_{1} I_{1}^{*}+E_{3} I_{3}^{*}+E_{5} I_{5}^{*}+U_{24} I_{g}^{*}=\mathrm{j} 7.0833[\mathrm{VA}] .
$$

The obtained values of the total complex power in expressions (32) and (33) are, as expected, equal.

Example 3 For the AC electrical network of figure 9 containing power sources $E_{1}=(10+\mathrm{j} 20)[\mathrm{V}]$, $E_{3}=(3-\mathrm{j} 11)[\mathrm{V}], E_{5}=(-3+\mathrm{j} 11)[\mathrm{V}], I_{g}=\mathrm{j} 0.5[\mathrm{~A}]$ and all impedances are mutually equal, e.g. $Z_{1}=Z_{2}=Z_{3}=Z_{4}=Z_{5}=(10+\mathrm{j} 20)[\Omega]$, iterative application of the proposed decomposition method results in $S_{E, N_{T}}=(5+\mathrm{j} 10)[\mathrm{VA}]$.

Example 4 For the $\mathrm{AC}$ electrical network of figure 9 containing power sources $E_{1}=(10+\mathrm{j} 20)[\mathrm{V}]$,
$E_{3}=(3-\mathrm{j} 11)[\mathrm{V}], E_{5}=(-3+\mathrm{j} 11)[\mathrm{V}], I_{g}=\mathrm{j} 0.5[\mathrm{~A}]$ and all impedances are resistive $Z_{1}=100[\Omega], Z_{2}=70[\Omega]$, $Z_{3}=30[\Omega], Z_{4}=20[\Omega]$ and $Z_{5}=80[\Omega]$, by the proposed iterative application of the proposed decomposition method one obtains $S_{E, N_{T}}=(13+\mathrm{j} 0)$ [VA].

\section{Conclusion}

The proposed approach for total power calculation is conceptually simple and computationally effective, indicating that the proposed decomposition of the complex network to simple networks needs smaller processing time for total power calculation. The proposed method allows iterative decomposition of a complex LEN. The total power dissipated by impedances of the starting network is equal to the sum of powers dissipated by impedances of the decomposed networks under the condition that all network impedances $Z$ are either resistive $\operatorname{Im}(Z)=0$ or reactive $\operatorname{Re}(Z)=0$ or mutually equal. A favourable feature of the proposed method is that it offers a flexible approach to any linear network, i.e., by application of the method at each subsequent iteration the network becomes simpler. The flexibility is related to the strategy in performing the basic steps of the method when analysing complex networks. In this way, one obtains a network consisting of simple elements involving calculations carried out through already developed algorithms. It is interesting to mention that in further investigations concerning the elaboration of the strategy of application of the method, it is justified that decomposition of networks should be considered from the optimization point of view, as well as the elimination of the limitations as regards impedances in linear electrical AC networks.

\section{Acknowledgements}

Author Tomislav B Šekara gratefully acknowledges support of the Ministry of Education, Science and Technological Development of the Republic of Serbia by Grant TR33020.

\section{References}

[1] Charles A D and Ernest S K 2009 Basic circuit theory. India: McGraw-Hill Education Pvt Limited

[2] Norman B and Theodore B 1981 Linear network theory: analysis, properties and synthesis. Beaverton, Oregon: Matrix Publishers Inc.

[3] Bessonov L A 2016 Basic electrical engineering theory: electric circuits, 12th edn. Moskva: Yurayt (in Russian)

[4] Ferreira L A F M 1992 Tellegen theorem and modelling of power systems-PV buses and nonlinear formulas. In: IEE Proceedings G-Circuits, Devices and Systems 139(1): 136-140. https://doi.org/10.1049/ip-g-2.1992.0023

[5] Vittal V and Michel A N 1988 A variational principle for nonconservative power systems. Circuits, Systems and Signal Processing 7(3): 413-424 
[6] Rácz L Z 1973 A graph theory approach of decomposition techniques in power system calculation. Periodica Polytechnica EL 17/3: 217-224

[7] Gupta H and Sharma J 1980 Decomposition techniques for circuit analysis. In: Proceedings of ESSCIRC: 6th European Solid State Circuits Conference, Grenoble, France, 22-25 September

[8] Demov O D and Palamarchuk O P 2009 Decomposition of electrical networks at calculation reactive power compensation in them. Visnyk Natsionalnoho Universytetu "Lvivska politekhnika". Elektroenerhetychni ta elektromekhanichni systemy (in Ukrainian), No. 637, p. 2427

[9] Demov O D and Pivniuk Y Y 2017 Step-by-step calculation of reactive power compensation in electrical networks based on their decomposition. Tekhnichna Elektrodynamika 1: 81-86 (Jan/Feb)

[10] Tomislav B Š 1995 A method of decomposition of power in linear electrical networks. In: Proceedings of the XXXIX ETRAN Conference (in Serbian), Zlatibor, 6-9.VI, pp. 319-322

[11] Chen W K 1990 A general n-port network reciprocity theorem. IEEE Transactions on Education 33(4): 360-362
[12] Penin A 2015 Analysis of electrical circuits with variable load regime parameters. Switzerland: Springer International Publishing AG.

[13] Jovan $\check{C}$ M and Tomislav B Š 2015 A new reactive power definition based on the minimization of the load non-reactive currents. In: Proceedings of the 2015 12th ConferenceSeminar of International School on Nonsinusoidal Currents and Compensation, Lagow, Poland, pp. 1-6. https://doi.org/ 10.1109/ISNCC.2015.7174683

[14] Czarnecki L S 2015 Currents' physical components (CPC) in four-wire systems with nonsinusoidal symmetrical voltage. Przeglad Elektrotechniczny 1(6): 50-55. https://doi.org/10. 15199/48.2015.06.07

[15] Tomislav B Š, Jovan Č M and Djurišic Ž R 2008 Optimal reactive compensators in power systems under asymmetrical and nonsinusoidal conditions. IEEE Transactions on Power Delivery 23(2): 974-984

[16] Hanoch L A, Aleksandar M S and Sergio J C 2005 An orthogonal decomposition of apparent power to application to an industrial load. In: Proceedings of the Power Systems Computation Conference (PSCC), Liege, Belgium, August, Session 22, Paper 5, pp. 1-7 